\title{
Enhancement of the Auditory Late Response (N1-P2) by Presentation of Stimuli From an Unexpected Location
}

DOI: $10.3766 /$ jaaa. 17047

\author{
Raquel M. Heacock* \\ Amanda Pigeon* \\ Gail Chermak $\dagger$ \\ Frank Musiek \\ Jeffrey Weihing*
}

\begin{abstract}
Background: Passive electrophysiological protocols, such as the middle latency response and speech auditory brainstem response, are often advocated in the objective assessment of central auditory processing disorder (CAPD). However, few established electrophysiological protocols exist for CAPD assessment that have patients participate in active tasks which more closely approximate real-world listening. To this end, the present study used a discrimination task (i.e., oddball paradigm) to measure an enhancement of the auditory late response (N1-P2) that occurs when participants direct their auditory attention toward speech arising from an unexpected spatial location.
\end{abstract}

Purpose: To establish whether N1-P2 is enhanced when auditory attention is directed toward an unexpected location during a two-word discrimination task. In addition, it was also investigated whether any enhancements in this response were contingent on the stimulus being counted as part of the oddball paradigm.

Research Design: Prospective study with a repeated measures design.

Study Sample: Ten normal hearing adults, with an age range of 18-24 years.

Data Collection and Analysis: The N1 and P2 latencies and peak-to-peak amplitudes were recorded during a P300 paradigm. A series of repeated measures of analysis of variance and a correlation analysis was performed.

Results: There was a significant effect of stimulus location, in which words arising from the unexpected location showed a larger N1-P2 peak-to-peak amplitude and an earlier N1 latency. This effect was seen regardless of whether or not participants had to count the word total in memory.

Conclusions: These findings suggest that spatial enhancement of the N1-P2 is a fairly robust phenomenon in normal hearing adult listeners. Additional studies are needed to determine whether this enhancement is absent or reduced in patients with CAPD.

Key Words: attention, auditory processing disorders, dichotic listening, electrophysiology, N1-P2, spatial processing

Abbreviations: ANOVA = analysis of variance; CAPD = central auditory processing disorder; EP = evoked potentials; SD = standard deviation; SEC = spatial enhancement component

${ }^{*}$ School of Medicine, University of Louisville, Louisville, KY; †Elson S. Floyd College of Medicine, Washington State University, Spokane, WA; $\ddagger$ School of Medicine, University of Arizona, Tuscon, AZ

Corresponding author: Jeffrey Weihing, Program in Audiology, MDA, University of Louisville, Louisville, KY 40292; Email: jaweih02@louisville.edu 


\section{INTRODUCTION}

$\mathrm{C}$ entral auditory processing is assessed in the clinic using behavioral measures that typically require patients to provide a verbal response. There has been increased interest in developing objective measures of auditory processing that assess the integrity of the central auditory nervous system without requiring that patients respond verbally (Strauss et al, 2004; Strait et al, 2011). These objective measures are often considered advantageous because they limit the requirements placed on the patient and, therefore, may be less affected by confounds such as poor speech-language and cognitive ability or motor skills. The more commonly used objective measures are electrophysiological responses recorded to auditory stimuli, such as the middle latency response (Musiek et al, 1984) and the complex auditory brainstem response (Skoe and Kraus, 2010).

Although electrophysiological measures designed to assess auditory processing are available for use in clinical practice, these paradigms are often passive in nature and do not necessarily involve listening abilities individuals use in real world environments. Patients are often instructed to sit still and listen, or watch a movie, while stimuli are presented to acquire the electrophysiological response. Electrophysiological measures that evaluate abilities that are more similar to those recruited during active listening may provide an alternative, potentially more ecologically valid, description of the abilities underlying auditory processing.

An electrophysiological paradigm that could more closely assess active listening is one that acquires evoked potentials (EPs) to directed auditory attention. Directing auditory attention toward acoustic events of interest is an important skill that benefits listening in noise (Shinn-Cunningham and Best, 2010). Typically this ability is assessed clinically using behavioral central auditory processing tests, such as dichotic listening (e.g., Competing Sentences test [Willeford et al, 1994]) or spatial processing measures (e.g., Listening in Spatialized Noise [Cameron et al, 2006]). In these tests, patients are asked to direct their attention toward a speech stimulus that arrives from a particular spatial location while ignoring a competing stimulus. An electrophysiological correlate might assess the directed-attention aspects of these behavioral paradigms by measuring the neuroelectric response that occurs when attention is directed to a spatial location that contains a stimulus of interest.

A variety of EPs could be used to assess directed auditory attention. The N1-P2 complex of the auditory late response is particularly well suited in this regard because this response is highly reliable (Roth et al,
1975; Walhovd and Fjell, 2002) and easily acquired with existing clinical equipment. In addition, the N1-P2 shows a measurable enhancement (i.e., increase in peak-to-peak amplitude) when participants are asked to attend-to the auditory stimulus, particularly when the stimulus is novel in some way (Davis, 1964; Spong et al, 1965; Picton et al, 1971; Hillyard et al, 1973). This enhancement has been shown to occur as a result of switching attention to auditory stimuli arriving from different locations. For instance, Okita and Ohtani (1977) reported on an N1-P2 paradigm in which participants listened to stimuli that came from a single fixed location, or required constant shifts in directed auditory attention because the stimuli came from different locations (i.e., left or right ear). They noted that the N1-P2 amplitude was significantly larger when participants were required to constantly shift their directed auditory attention toward different locations.

An objective measure of directed auditory attention could be useful in identifying auditory processing difficulties in patients seen for listening difficulties. In the present study, we report on a clinically feasible paradigm for measuring the electrophysiological response that arises when normal hearing adult participants direct their attention toward a spoken word that arises from an unexpected location. The paradigm is based primarily on earlier research from our laboratory that tended to show an enhancement of the N1-P2 peakto-peak amplitude when participants attended to a stimulus of this type (Pigeon et al, 2016). In the paradigm used in the present study, participants heard two different words over earphones and were tasked to attend-to one of them. The task required that they attend-to and count (silently remember) the specified target word while ignoring the nontarget word. Both words occurred more frequently in the right ear (i.e., expected location), but would sometimes be presented in the left ear (i.e., unexpected location). Responses obtained from the expected and unexpected location were then compared.

Specifically, the present study was constructed with three study aims in mind. The first was to determine if there is an enhancement of the auditory N1-P2 when participants are asked to direct their attention to the target word arising from the unexpected location, and second to determine if it is necessary for participants to perform a task with the stimulus (i.e., count the target word in memory) to obtain this enhancement, or whether the enhancement is obtained for the ignored nontarget word as well. The third aim of the study was to determine if the latency characteristics of the N1-P2 are affected by the location of the stimuli. Based on results from Pigeon et al (2016), the N1-P2 was expected to be enhanced because of the interaction of stimulus type and location, or to be enhanced both by type (target) and location (unexpected). 


\section{METHODS}

\section{Subjects}

Ten normal hearing male and female participants participated in the present study (age range $=18-25$, mean $=22.8$ years, standard deviation $[\mathrm{SD}]=1.887$ ). All participants showed normal peripheral hearing, defined as: pure tone air conduction thresholds $\leq 20 \mathrm{~dB}$ HL for all octave frequencies between 500 and 4000 $\mathrm{Hz}$ bilaterally, and normal type A tympanograms in both ears. Participants had no reported neurological issues. Participants also passed a central hearing screening, obtaining a score of $\geq 90 \%$ in each ear on the Dichotic Digits Test (Musiek, 1983). Two additional participants were excluded from the study because of excessive noise present during electrophysiological testing.

\section{Equipment}

The N1-P2 EP response was recorded using an Intelligent Hearing Systems Smart EP unit. Impedance was maintained at $3 \mathrm{kOhms}$ or less for each individual electrode. The recording time window was $512 \mathrm{msec}$. A 1-30 $\mathrm{Hz}$ analog filter was applied online along with a voltagedependent artifact reject filter. A 2012 HP ProBook $6565 \mathrm{~b}$ laptop was used for the recording. The laptop was connected to a grounded power source at all times. The audio signal was presented through the Intelligent Hearing Systems unit via insert earphones.

Electrodes were placed at $\mathrm{Cz}, \mathrm{A} 1, \mathrm{~A} 2$, and the right outer canthus. The outer canthus electrode was used for monitoring eye blinks. The ground electrode was located at the forehead. Evoked responses were recorded simultaneously from A1 and A2 and the recordings at each site were averaged together so that an average reference response could be computed that was not biased toward either ear. The present study recorded only from $\mathrm{Cz}$, as a previous study recorded from both $\mathrm{Cz}$ and $\mathrm{Pz}$, showing effects at both sites (Pigeon et al, 2016). As Cz is more primarily used in the clinic, this approach was used to support clinic feasibility.

\section{Stimulus}

A P300 oddball paradigm (Hillyard et al, 1973) was used for this study. Participants attended to two naturally recorded consonant vowel consonant words (i.e., "dog" and "cat") spoken by a male talker. One word served as the rare target word to which participants were instructed to count. The other word served as the frequent nontarget word, which participants were asked to ignore. The target word (either "dog" or "cat") was counterbalanced across participants. Each word was approximately $550 \mathrm{msec}$ in duration. Stimuli were presented monotically via insert earphones to the left and right ears during the study. The nontarget word was presented $52 \%$ of the time in the right ear and $16 \%$ of the time in the left ear $(68 \%$ total for the nontarget word), whereas the target word was presented $16 \%$ of the time in the right ear and $16 \%$ of the time in the left ear (32\% total for target word). In addition, stimuli occurred more often in the expected location at the right side ( $52 \%$ for nontarget words in the right ear $+16 \%$ for target words in the right ear) than from the unexpected location at the left side (16\% for nontarget words in the left ear $+16 \%$ for target words in the right ear). In an oddball paradigm, stimuli in one or more conditions are presented less frequently than the other conditions to evoke an enhanced electrophysiological response to the less frequently occurring stimuli. This enhancement typically indicates successful discrimination by the central auditory nervous system of the difference between the stimuli. This is why the paradigm was designed so that target words occurred less often than nontarget words, and stimuli occurring from the unexpected location occurred less often than stimuli occurring from the expected location. Figure 1 provides an example of how stimuli were presented in the present study.

\section{Procedure}

Testing was performed in a sound-treated booth. Participants were instructed to keep their body steady and look straight ahead at a picture to control for muscle artifact during testing. Participants were told that they would hear the words "dog" and "cat" and that they should count the number of times they heard the target word and ignore the nontarget word. Accuracy was evaluated after the run by asking them how many times they heard the target words. Stimuli were presented at $80 \mathrm{dBnHL}$ at a rate of $1.10 / \mathrm{sec}$. The reference thresholds for the stimuli were determined by computing the average behavioral thresholds to the stimuli obtained from five normal hearing listeners. Before collecting electrophysiological data that addressed the study questions, 100 stimuli were presented in the right ear only, $68 \%$ being nontarget words and $32 \%$ being target words. This condition was included to prime the right ear so as to create in an expectation of hearing words on this side/ear. These results were not analyzed.

For the experiment proper, three runs of 100 stimuli were presented, distributed as follows: $52 \%$ nontarget words in the expected location, $16 \%$ target words in the expected location, $16 \%$ nontarget words in the unexpected location, and $16 \%$ target words in the unexpected location. Waveforms for all three runs were averaged together for the latter three conditions, yielding a total of 48 trials per condition (16 trials $\times$ three runs). For the nontarget word expected location condition, only one run of 52 trials was used. This approach 


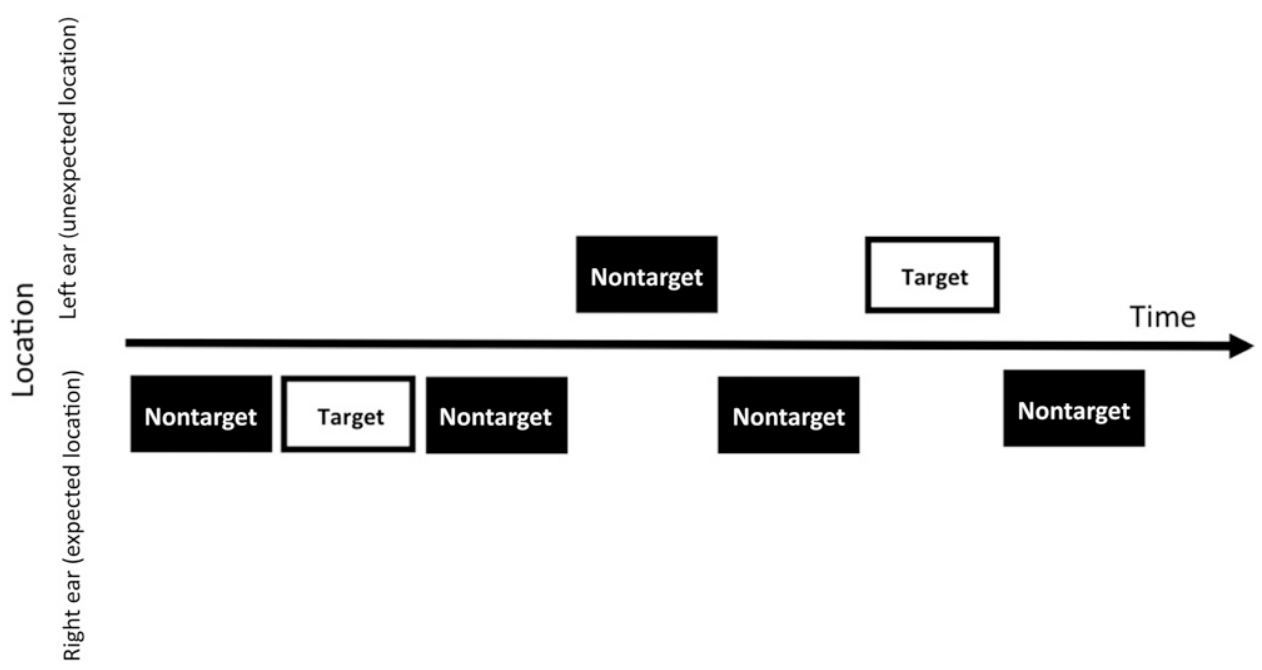

Figure 1. Stimulus representation for both the target and nontarget word to the expected (right) and unexpected (left) ears.

was adopted so that all conditions would have a similar number of trials contributing to the waveforms (i.e., 52 trials in the nontarget word expected location condition and 48 trials in all other conditions). The nontarget word expected location waveform selected for analysis from the three runs was based on which waveform showed the best and most similar morphological characteristics for N1 and P2. Two of the present authors (R.H. and J.W.) evaluated the waveforms separately and agreed on which run to use in the data analysis. Both authors were familiar with the morphology of this response and had experience with peak picking on this particular equipment. The authors made these judgments independently and then discussed their selections. Differences between evaluators were rare. In cases where this occurred, all possible pairings of waveforms were considered until two were chosen that both evaluators agreed on. Emphasis was placed on which two waveforms yielded the best and most similar morphology for N1-P2.

\section{Computation of Difference Waveform}

A difference waveform was computed for each participant to highlight any potential enhancement in N1-P2 amplitude that would occur by attending to the word at the unexpected location. This waveform, calculated separately for the target and nontarget words, represented the difference between the unexpected location EP waveform and the expected location EP waveform. A large positivity in this difference waveform represents an increased neural response when the word arose from the unexpected location. We refer to this positivity in the difference waveform as the spatial enhancement component (SEC). The SEC was computed for some of the following analyses. The amplitude of the SEC was measured as the peak-to-peak amplitude from the maximum of the SEC peak to the preceding trough.

\section{Statistical Analysis}

A series of repeated measures of analysis of variance (ANOVA) were performed. The mean target word N1P2 amplitudes occurring at the expected and unexpected locations were compared to determine whether the unexpected word elicited an amplitude enhancement. The mean nontarget word amplitude also was examined in a similar manner to determine whether the amplitude enhancement also occurred in the absence of the oddball paradigm counting task. A third ANOVA examined whether the location and stimulus factors had any effect on the latency of the N1 or P2.

\section{RESULTS}

\section{N1-P2 Amplitude Effects}

Descriptive statistics for the N1-P2 amplitudes are shown in Figure 2. A repeated measures ANOVA was conducted for the dependent measure N1-P2

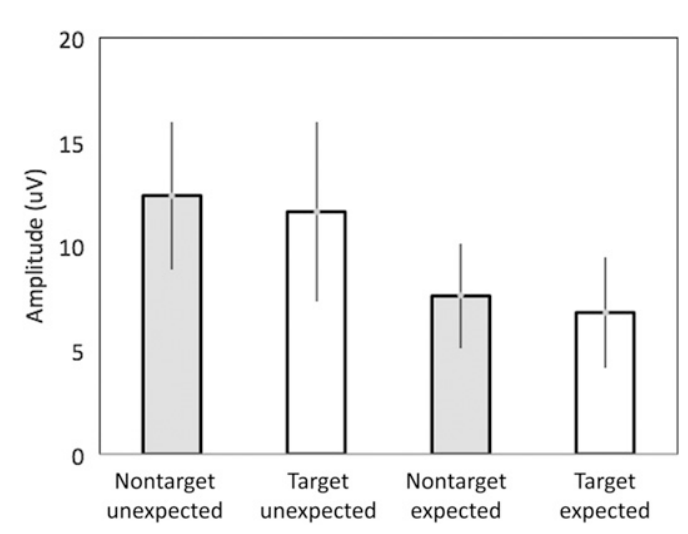

Figure 2. N1 P2 peak-to-peak amplitude for all of the conditions in the present study. One SD error bar shown. 
peak-to-peak amplitude using factors: stimulus type (target versus nontarget) and stimulus location (expected versus unexpected location). A significant main effect was observed for stimulus location $\left[F_{(1,9)}=39.69\right.$, $p<0.001$, whereas a nonsignificant main effect of stimulus type $\left[F_{(1,9)}=1.01, p=0.34\right]$ and a nonsignificant interaction of stimulus location and type $\left[F_{(1,9)}=0.01\right.$, $p=0.97]$ were noted. The N1-P2 peak-to-peak amplitude increased when the stimulus originated from an unexpected location and this location enhancement was noted for both the target and nontarget words.

\section{N1 and P2 Latency Effects}

Descriptive statistics for $\mathrm{N} 1$ and P2 latencies are depicted in Figures 3 and 4, respectively. A repeated measures ANOVA was conducted for the dependent measures N1 and P2 latency using factors: stimulus type (target versus nontarget) and stimulus location (expected versus unexpected location). For N1, a significant main effect was observed for stimulus location $\left[F_{(1,9)}=9.06, p<0.02\right]$, whereas a nonsignificant main effect of stimulus type $\left[F_{(1,9)}=0.75, p=0.41\right]$ and a nonsignificant interaction of stimulus location and type $\left[F_{(1,9)}=0.28, p=0.61\right]$ were noted. For P2, nonsignificant main effects were observed for both stimulus location $\left[F_{(1,9)}=0.95, p=0.36\right]$ and stimulus type $\left[F_{(1,9)}=\right.$ $1.30, p=0.28]$, as well as for the interaction of stimulus location and type $\left[F_{(1,9)}=0.93, p=0.36\right]$. This series of ANOVAs confirms that N1 latency occurred earlier when the stimulus arrived from the unexpected location for both the target and nontarget word, whereas no significant latency effects were noted for P2.

\section{Description of the SEC}

Grand average waveforms for the target and nontarget words, and for the SEC, are depicted in Figures 5 and 6 . Figure 6 also shows the grand average of the SEC along with the individual SEC responses for all

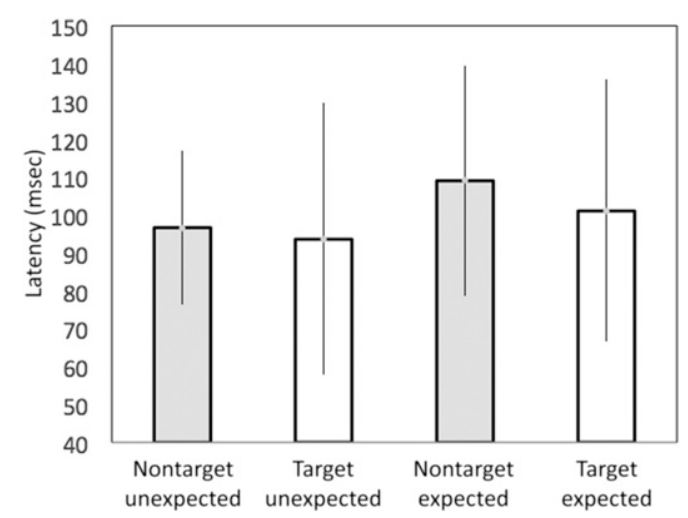

Figure 3. N1 latencies for all of the conditions in the present study. One SD error bar shown.

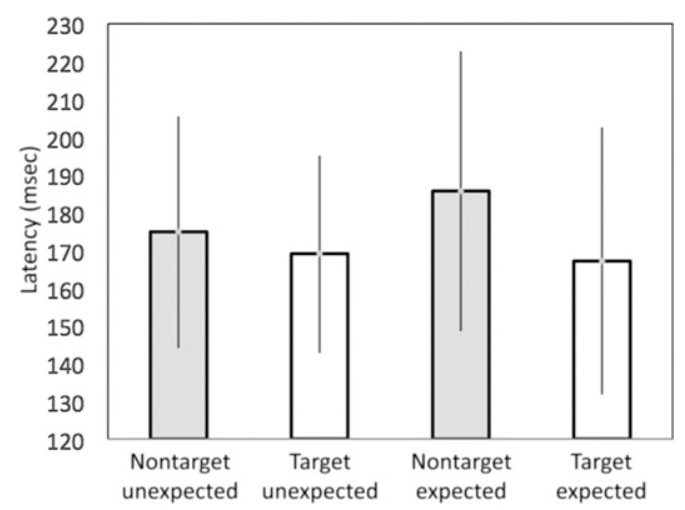

Figure 4. P2 latencies for all of the conditions in the present study. One SD error bar shown.

ten participants. Individual participant responses have been normalized in Figure 6 so that their maximum peak voltage is equal to the peak voltage of the grand average waveform. Examined qualitatively, it is clear that most participants showed the SEC, indicating that their response to stimuli at the unexpected location was higher in amplitude than their response to stimuli at the expected location. The mean amplitude of the SEC for the nontarget waveform was $9.35 \mu \mathrm{V}$ with an SD of 2.94, whereas mean amplitude of the target waveform was $8.66 \mathrm{uV}$ with an SD of 2.61. The mean latency of the SEC peak for the nontarget waveform was $167.20 \mathrm{msec}$ with an SD of 26.57, whereas the latency of the SEC peak for the target waveform was 162.30 msec with an SD of 17.65. Therefore, it appears that this enhancement effect was present regardless of stimulus type (target or nontarget) or whether participants ignored the stimulus or performed the counting task. As seen in both Figure 6 and in the SDs of SEC latency, there appeared to be less between-subject variability in the latency of the target SEC when compared with the nontarget SEC.

\section{DISCUSSION}

$\mathrm{T}$ he present study evaluated a new paradigm for assessment of directed auditory attention. Participants listened to a target and a nontarget word in both a frequently occurring expected location and an infrequently occurring unexpected location. Findings revealed an increase in the peak-to-peak amplitude of N1-P2 when participants attended to or counted the target word from the unexpected location. Interestingly, a significant enhancement of N1-P2 amplitude also was seen for the nontarget word when it was presented from the unexpected location. Taken together, these findings suggest unexpected stimulus location can yield a measurable electrophysiological enhancement, but that this increased response occurs regardless of whether or not the stimulus is task relevant (i.e., counting silently and retaining the target word in memory). 

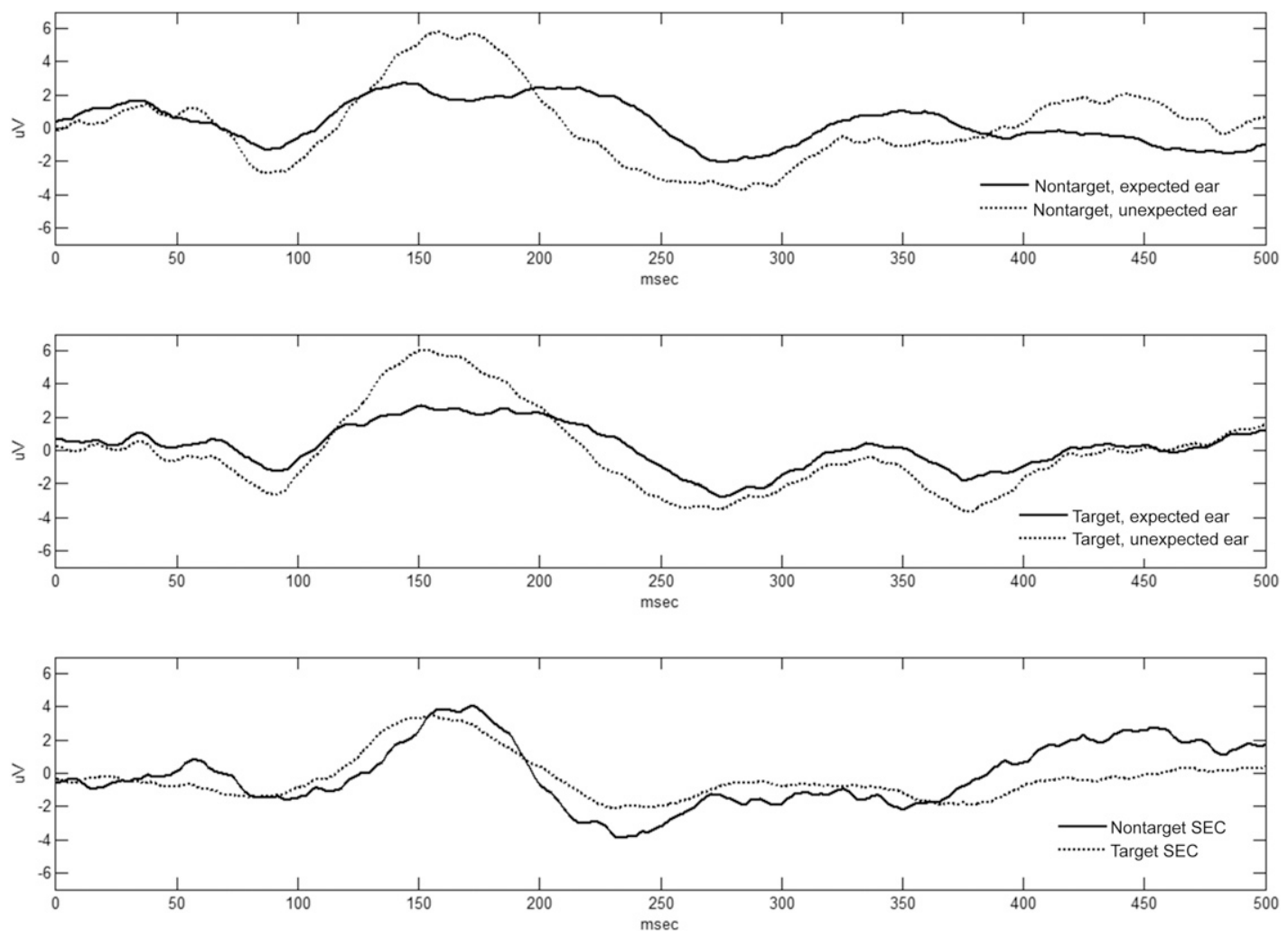

Figure 5. Grand average waveforms for all ten participants in the present study. One SD error bar shown.

This significant enhancement to stimulus arising from an unexpected location can be compared most directly with our earlier study that used a similar paradigm with normal hearing young adults in which we reported the same effect (Pigeon et al, 2016). It should be noted, however, that there were several methodological differences between the present study and Pigeon et al. Whereas the present study recorded only from $\mathrm{Cz}$, Pigeon et al recorded from both $\mathrm{Cz}$ and $\mathrm{Pz}$, showing effects at both sites. The stimuli and paradigm also vary across studies: Pigeon et al used nonspeech stimuli, whereas the present study used speech and the present study presented both the target and nontarget words at the unexpected location whereas Pigeon et al presented only the target word at this location. Nevertheless, the finding that this enhancement occurs regardless of the stimulus and paradigm type speaks to the robust nature of this finding.

Our location effect was also similar to the results of Okita and Ohtani (1977) who examined the effect of switching attention between ears on the N1-P2, showing that when participants switched attention between ears during an EP paradigm, the resulting N1-P2 was larger in amplitude than when attention was only directed to a single ear. These findings are consistent with the results of the present study, which showed an enhancement of N1-P2 when participants directed attention to a stimulus arising from an unexpected location (i.e., attention "switched" to the contralateral ear). There are several key differences between the paradigm used by Okita and Ohtani and the present study. The present study required participants to perform a discrimination task (e.g., count the target word), whereas Okita and Ohtani presented only a single stimulus for participants count it. In addition, the present study recorded separate waveforms for the left and right ears, whereas Okita and Ohtani recorded responses from each ear in the same waveform. Nonetheless, the findings across these two studies are remarkably similar despite the procedural differences.

Enhancement of the N1-P2 as a result of increased attention has been well documented in the literature, although most studies have not examined this measure in response to changes in stimulus spatial location. Hillyard et al (1973) presented two rapid trains of stimuli simultaneously to participants' left and right ears. When asked to perform a discrimination task by attending to either the left or right ear, the N1-P2 was larger in the ear to which participants attended and smaller in the contralateral ear. Davis (1964) also observed this enhancement and noted that it was more likely if the auditory discrimination task was made more challenging. Picton et al (1971) noted larger evoked responses when participants attended to the auditory stimulus as opposed to reading during stimulus presentation. Spong et al (1965) who recorded 

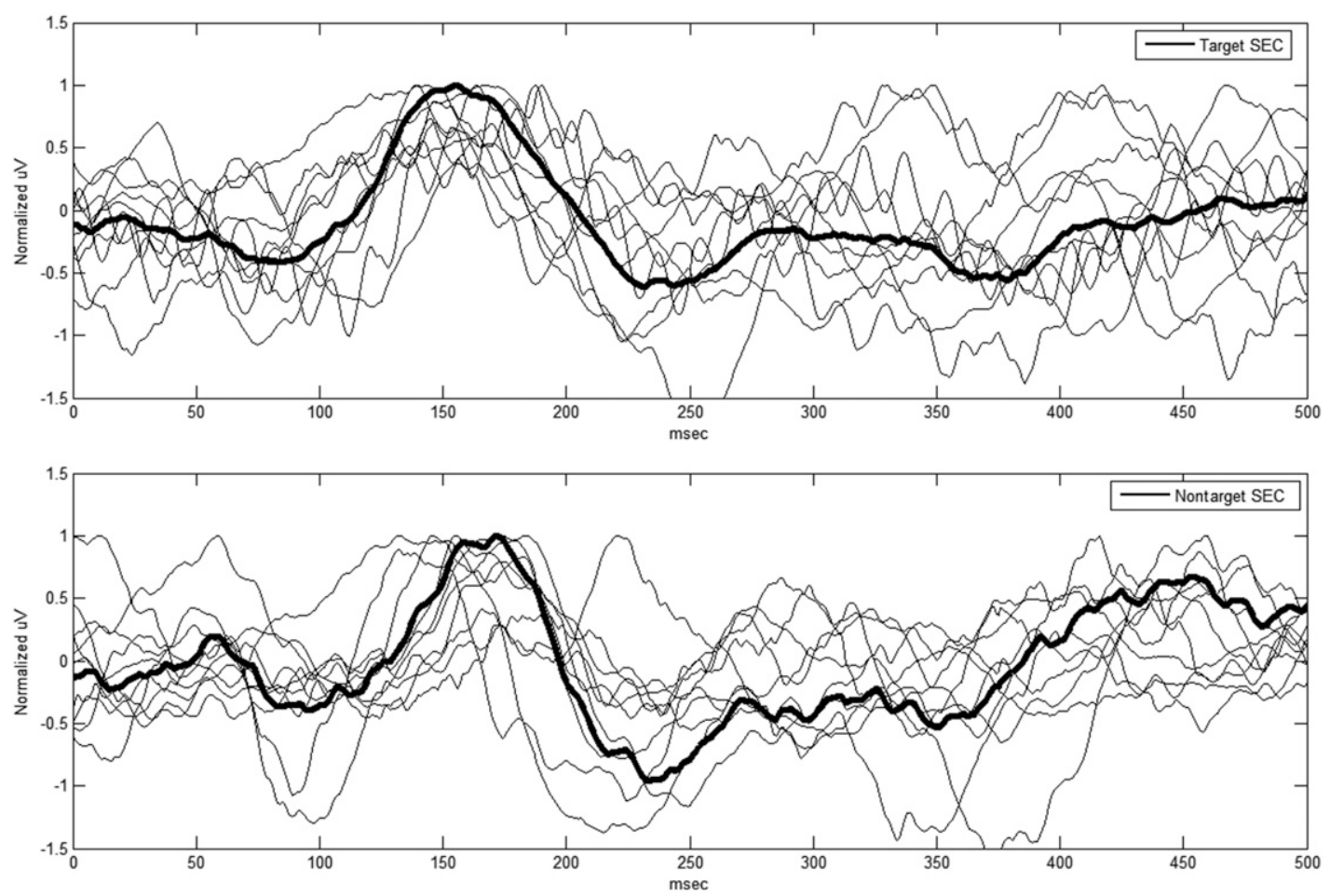

Figure 6. Individual (thin lines) and grand average waveforms (thick line) for all ten participants for the target word and nontarget word-unexpected location conditions.

visual and auditory EPs when participants were performing either a visual or auditory task, showed larger auditory evoked responses when performing the auditory task than when performing the visual task. The findings across studies suggest that the enhancement of N1-P2 in the present study may indicate that the occurrence of the word from the unexpected location momentarily alerts attention to that side (i.e., directed auditory attention diverts to the unexpected side).

Of interest is that the stimulus location effect was significant, whereas the stimulus type effect was not. There are several possibilities as to why this enhancement was noted in response to both the target and nontarget stimuli. It is possible that when the stimulus arises from the unexpected location it is alerting enough that it yields an enhanced N1-P2, regardless of whether the stimulus is counted or not. It is also possible that even though the nontarget word was not explicitly part of the task, it still required attention to perform the task accurately. Independent of the underlying mechanism, the results suggest that the N1-P2 enhancement is not contingent on the counting and memory aspects of the oddball paradigm because both stimuli evoked an enhancement yet only the target stimulus used these skills.

The degree of N1-P2 enhancement obtained with this paradigm is best demonstrated by examination of the "unexpected minus expected location" difference waveform. As shown in Figures 5 and 6, the SEC shows a positive component in the vicinity of $160 \mathrm{msec}$ that re- flects the degree of N1-P2 enhancement obtained by directing attention to the unexpected location. The SEC was fairly robust (8-9 $\mu \mathrm{V}$ on average) and all participants demonstrated a component in this region. It is possible that using the amplitude of this component to quantify the results of this measure may prove diagnostically useful as it may best describe the properties being targeted by this paradigm.

Compared with the amplitude findings, the effects of stimulus location on the absolute latencies of $\mathrm{N} 1$ and $\mathrm{P} 2$ were less notable. The only significant effect observed was that of the location factor on the N1 latency, where stimuli from the unexpected location elicited earlier latencies. The enhancement of N1-P2 that occurs when stimuli arise from the unexpected location also appears to delay the P2 latency relative to the expected location condition, at least when the trends are considered. In other words, the larger amplitude N1-P2 response that occurred as a result of the unexpected location stimulus also tends to push out the latency of the $\mathrm{P} 2$ peak. Therefore, this later P2 latency may be a consequence of the increased neural response to the stimulus arising from the unexpected location. Although this finding is encouraging, it requires replication in a larger sample.

\section{Future Directions}

It is possible that measurements of the waveform from the unexpected location or the SEC obtained from 
the "unexpected minus expected location" difference waveform may be diagnostically useful in separating individuals with and without certain types of central auditory processing disorder (CAPD). As this study did not recruit patients with CAPD, a follow-up study investigating clinical populations would be necessary to establish whether the paradigm in general is diagnostically useful and what indices best separate patients with and without the disorder.

Future studies might also incorporate a larger number of participants. The sample used in the present study was large enough to identify effects because a significant difference was noted for the stimulus location variable. Furthermore, these same findings were noted across both the present study and an earlier study conducted by Pigeon et al (2016). That being said, it is possible that a larger sample might identify additional significant effects for the stimulus type manipulation. It should be noted that overall, the difference between conditions for this variable were quite small and, even if significant, it is questionable whether the difference would be large enough to be meaningful.

\section{CONCLUSIONS}

$\mathrm{T}$ he present study demonstrated that the N1-P2 is enhanced when hearing a word arise from an unexpected location. This enhancement was unrelated to whether or not the word was targeted (i.e., counted). Given past findings that demonstrate that the N1-P2 is enhanced by attention (Davis, 1964; Spong et al, 1965; Picton et al, 1971; Hillyard et al, 1973), it is possible that this increase in N1-P2 amplitude arises from attention being directed toward the unexpected location. Future research should determine whether this enhancement differentiates individuals with CAPD from those with normal central auditory function.

\section{REFERENCES}

Cameron S, Dillon H, Newall P. (2006) Development and evaluation of the listening in spatialized noise test. Ear Hear 27:30-42.
Davis H. (1964) Enhancement of evoked cortical potentials in humans related to a task requiring a decision. Science 14:182-183.

Hillyard SA, Hink RF, Schwent VL, Picton TW. (1973) Electrical signs of selective attention in the human brain. Science 182: $177-180$

Musiek FE. (1983) Assessment of central auditory dysfunction: the dichotic digit test revisited. Ear Hear 4:79-83.

Musiek FE, Geurkink NA, Weider DJ, Donnelly K. (1984) Past, present, and future applications of the auditory middle latency response. Laryngoscope 94:1545-1553.

Okita T, Ohtani A. (1977) The effects of active attention switching between the ears on averaged evoked potentials. Electroencephalogr Clin Neurophysiol 42:198-204.

Picton TW, Hillyard SA, Galambos R, Schiff M. (1971) Human auditory attention: a central or peripheral process? Science 173: 351-353.

Pigeon A, Miller S, Weihing J. (2016) An Electrophysiological Measure of Attention Switching. Phoenix, AZ: American Academy of Audiology Conference.

Roth W, Kopell B, Tinklenberg J, Huntsberger G, Kraemer H. (1975) Reliability of the contingent negative variation and the auditory evoked potential. Electroencephalogr Clin Neurophysiol 38: $45-50$.

Shinn-Cunningham BG, Best V. (2008) Selective attention in normal and impaired hearing. Trends Amplif 12:283-299.

Skoe E, Kraus N. (2010) Auditory brainstem response to complex sounds: a tutorial. Ear Hear 31:302.

Spong P, Haider M, Lindsley DB. (1965) Selective attentiveness and cortical evoked responses to visual and auditory stimuli. Science 148:395-397.

Strait DL, Hornickel J, Kraus N. (2011) Subcortical processing of speech regularities underlies reading and music aptitude in children. Behav Brain Funct 7:44.

Strauss DJ, Delb W, Plinkert PK. (2004) Analysis and detection of binaural interaction in auditory evoked brainstem responses by time-scale representations. Comput Biol Med 34:461-477.

Walhovd KB, Fjell AM. (2002) One-year test-retest reliability of auditory ERPs in young and old adults. Int $J$ Psychophysiol 46: $29-40$.

Willeford J, Burleigh J, Katz J. (1994) Sentence procedures in central testing. Handb Clin Audiol 4:256-268. 